\title{
OFDM System Analysis for reduction of Inter symbol Interference Using the AWGN Channel Platform
}

\author{
D.M. Bappy, Ajoy Kumar Dey, Susmita Saha \\ Department of Signal Processing \\ Blekinge Tekniska Hogskola (BTH) \\ Karlskrona, Sweden \\ E-mail: dey.ajoykumar@yahoo.com
}

\author{
Avijit Saha, Shibani Ghosh \\ Department of Electrical and Electronic Engineering \\ Bangladesh University of Engineering and Technology \\ Dhaka, Bangladesh \\ E-mail: avijit003@gmail.com
}

\begin{abstract}
Orthogonal Frequency Division Multiplexing (OFDM) transmissions are emerging as important modulation technique because of its capacity of ensuring high level of robustness against any interferences. This project is mainly concerned with how well OFDM system performs in reducing the Inter Symbol Interference (ISI) when the transmission is made over an Additive White Gaussian Noise (AWGN) channel. When OFDM is considered as a low symbol rate and a long symbol duration modulation scheme, it is sensible to insert a guard interval between the OFDM symbols for the purpose of eliminating the effect of ISI with the increase of Signal to Noise Ratio (SNR).
\end{abstract}

Keywords- OFDM; Inter symbol Interference; AWGN; matlab; algorithm.

\section{INTRODUCTION (HEADING 1)}

This paper is intended for those engineers whose research interests are related to digital communication, particularly the terrestrial digital video broadcasting (DVB-T) and the digital audio broadcasting (DAB) as Orthogonal Frequency Division Multiplexing(OFDM) system has been effectively used in both digital television and radio broadcasting system. The OFDM usually operates with high data rate and hence it is also widely used in asymmetric digital subscriber lines (ADSL) in order to achieve a high speed data connection. Considering the cost as a factor, this paper excludes the need of an equalizer and shows that it is still possible to reduce the ISI in an OFDM system without the equalizer[6].

This project investigates the OFDM system performance in eliminating the Inter symbol Interference (ISI) effect which, in our experiment, is caused by the noise generated from the AWGN channel. In the case of using the single carrier modulation, frequency selective fading and inter symbol interference occur, which leads to high probability of errors, consequently, affecting on the system performance. So, it is crucially important to study the elimination process of ISI effect. Additionally, in our project, we have considered OFDM system because OFDM is a form of digital multi-carrier modulation method that is widely used in wireless communication in order to solve the problem. In order to reducing the ISI effects, a 16-QAM modulation technique and an AWGN channel are used in our experiment.

\section{LITERATURE REVIEW}

Assuming the ideal channel or noiseless environment, we do so in order to focus attention on the effects of imperfections in the frequency response of the channel, on data transmission through the channel[4].

The receiving filter output, we may be written as

$$
y(t)=\mu \sum_{k=-x}^{x} A_{k} p\left(t-k T_{b}\right)
$$

where $\mu$, is a scaling factor. The pulse $\mathrm{p}(\mathrm{t})$ has a shape different from that of $\mathrm{g}(\mathrm{t})$, but it is normalized such that,

$$
\mathrm{p}(0)=1 \text {. }
$$

The pulse $\mu A_{k} p(t)$ is the response of the cascade connection of the transmitting filter, the channel, and receiving filter, which is produced by the pulse $A_{k} g(t)$ applied to the input of this cascade connection. Therefore, we may relate $p(t)$ to $g(t)$ in the frequency domain as follows (after cancelling the common factor $A_{k}$ )

$$
\mu \mathrm{P}(\mathrm{f})=\mathrm{G}(\mathrm{f}) \mathrm{H}_{\mathrm{T}}(\mathrm{f}) \mathrm{H}_{\mathrm{C}}(\mathrm{f}) \mathrm{H}_{\mathrm{R}}(\mathrm{f})
$$

where $P(f)$ and $G(f)$ are the Fourier transform of $p(t)$ and $g(t)$, respectively. The receiving filter output $y(t)$ is sampled at time $\mathrm{t}_{\mathrm{i}}=i \mathrm{~T}_{\mathrm{b}}$ (with $i$ taking on integer values), yielding

$$
\begin{gathered}
\mathrm{y}\left(\mathrm{t}_{\mathrm{i}}\right)=\mu \sum_{\mathrm{k}=-\mathrm{s}}^{\mathrm{s}} \mathrm{A}_{\mathrm{k}} \mathrm{p}\left[(\mathrm{i}-\mathrm{k}) \mathrm{T}_{\mathrm{b}}\right] \\
\mu \mathrm{A}_{\mathrm{i}}+\mu \sum_{\substack{\mathrm{k}=-\mathrm{ks} \\
\mathrm{k} \neq \mathrm{i}}}^{\mathrm{se}} \mathrm{A}_{\mathrm{k}} \mathrm{p}\left[(\mathrm{i}-\mathrm{k}) \mathrm{T}_{\mathrm{b}}\right] \mathrm{i}=0, \pm 1_{x} \pm 2 \\
=
\end{gathered}
$$

In Equation 3, the first term $\mu A_{\mathrm{i}}$ represents the contribution of the $i$ th transmitted bit. The second term represents the residual effect of all other transmitted bits on the decoding of the $i$ th received bit; this residual effect is called inter symbol 
interference (ISI). In the absence of ISI, we observe from the equation 3 that

Figure 1. Baseband Transmission System

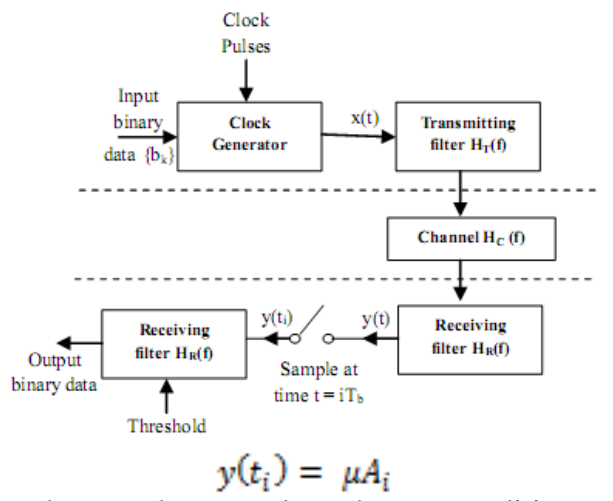

Which shows that, under these conditions, the $i$ th transmitted bit can be decoded correctly. The unavoidable presence of ISI in the system, however, introduces errors in the decision device at the receiver output. Therefore, in the design of the transmitting and receiving filters, the objective is to minimize the effects of ISI, and thereby deliver the digital data to its destinations with the smallest error rate.

Two previous researches demonstrate that ISI can be handled effectively in OFDM system by using the 4-QAM technique [1] [2]. ISI effect can be reduced by inserting a guard interval at low symbol rate instead of using pulse shaping filter at high symbol rate and this idea is drawn from [3].

\section{RESEARCH RATIONALE}

In an OFDM system with a higher symbol rate, reflected signals can interfere with consequent symbols. It creates complexity as the received signal can be distorted by this interference. So our research question arises, "how can we reduce ISI in an OFDM system?',

In the OFDM system we can mitigate this problem by modulating multiple sub-carriers per channel at lower symbol rate (long symbol period). By using a smaller symbol rate, the signal reflections are found occupying only a small portion of the total symbol period. Thus, it is possible to just insert a guard interval between consecutive OFDM symbols in order to remove interference from reflections. And then it becomes feasible for ISI to be removed from signals by increasing the value of SNR [5].

The main contributions of this project are:

1. Designing a new method that reduces the ISI in OFDM system very effectively. The method uses a guard interval which is equal to one-fourth of the symbol period, and also which includes a 16-QAM slicer, the slicer which is mainly used to compare the output constellation.

2. Implementing the whole model on MATLAB Workspace. In order to validate our experiment, the result of the simulation is shown in a plot of the SNR versus SER (Symbol Error Rate).

\section{ANALYSIS OF FINDINGS}

\section{A. OFDM signal generation and reception over AWGN channel}

The system model of the project is shown in Fig 1 where the upper part is the transmitter block and the lower part is the receiver block.

After working with the transmitter block, we have the transmitted signal $s(t)$ with the carrier frequency $f_{c}$,

$$
s(t)=u_{0} f_{1}(t) \cos \left(2 \pi f_{c} t\right)+u_{0} f_{Q}(t) \sin \left(2 \pi f_{c} t\right)
$$

From (1), the quadrature signal accords with the imaginary part of the complex modulation symbols and in our project, these are the 16-QAM symbols. The signal is then transmitted through an AWGN channel and the purpose of using this channel is to add a noise $n(t)$ to the transmitted signal $s(t)$. In the receiver part, the corrupted OFDM signal is first filtered by using a low pass filter in order to get the original baseband signal and then sampled. Finally, the output from FFT modulation gives the received constellation. The received constellation passes through a 16-QAM slicer and inserts the received symbols into the sixteen possible constellation points.

\section{B. ISI analysis and Validation}

In our experiment, we have considered only one path (AWGN channel) between OFDM transmitter and receiver which corrupts the transmitted signal. After getting the output from FFT block, it is possible to make a comparison between the original and received 16-QAM constellation. By observing the received constellation, a large amount of symbol errors arises which leads to high noise power, consequently, generates the ISI in the system. By increasing the value of SNR up to a certain level, we can reduce the Symbol Error Rate (SER) as well as the ISI. SNR.

Table 1 presents the symbol errors for different values of

Fig 3 shows the ideal 16QAM constellation that we get in the transmitter part. Fig 4 shows that the noise power and the ISI are found higher when the SNR value is fixed at $0 \mathrm{~dB}$. With the increase of SNR, ISI is found to be reduced effectively and this is shown in Fig 5. Finally, Fig 6 illustrates the system performance as good as we expected.

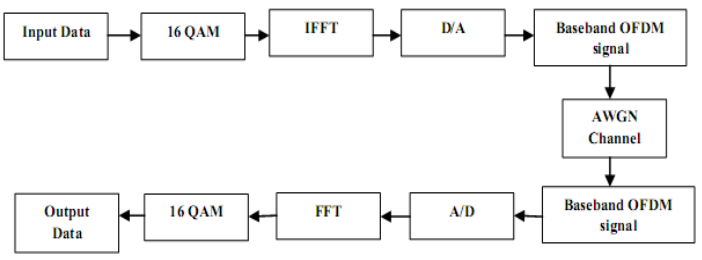

Figure 2. OFDM system model for 16-QAM 
TABLE 1. NO. OF SYMBOL ERRORS FOR DIFFERENT SNR

\begin{tabular}{|c|c|c|c|}
\hline SNR[dB] & Symbol Error & SNR[dB] & Symbol Error \\
\hline 0 & 612 & 12 & 44 \\
\hline 4 & 362 & 14 & 12 \\
\hline 8 & 203 & 16 & 5 \\
\hline
\end{tabular}

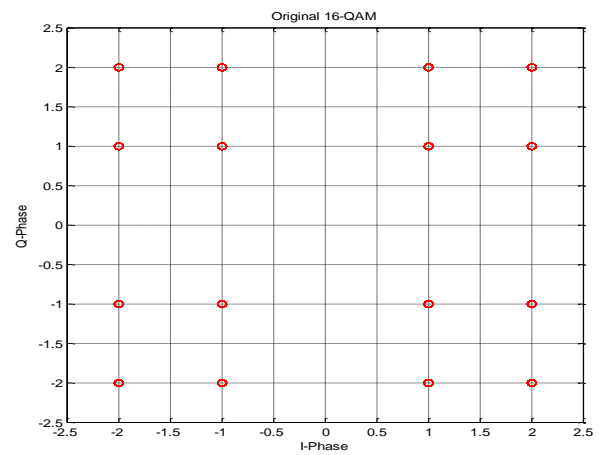

Figure 3. Original 16-QAM constellation

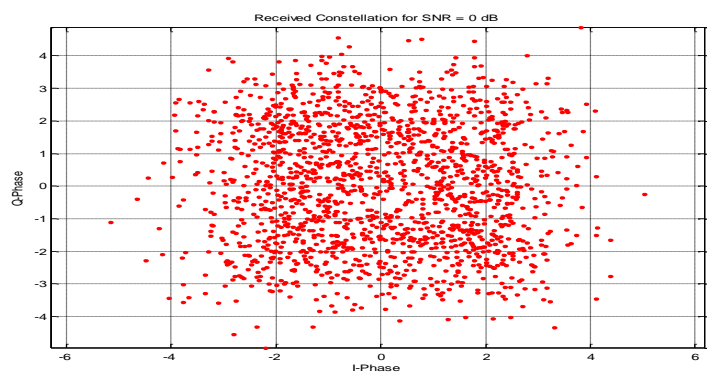

Figure 4. Received 16-QAM constellation with SNR $=0 \mathrm{~dB}$

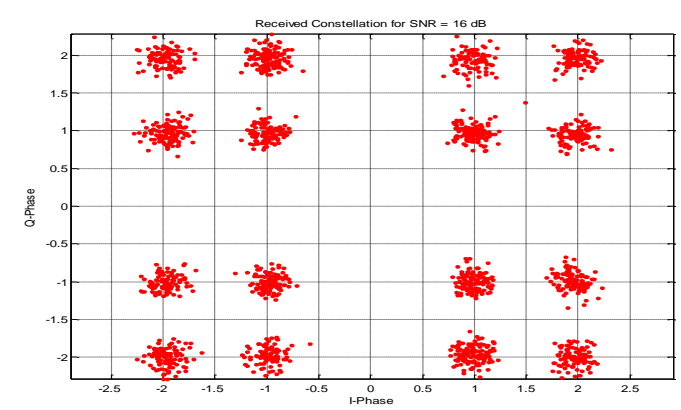

Figure 5. Received 16-QAM constellation with $\mathrm{SNR}=16 \mathrm{~dB}$

\section{CONCLUSION}

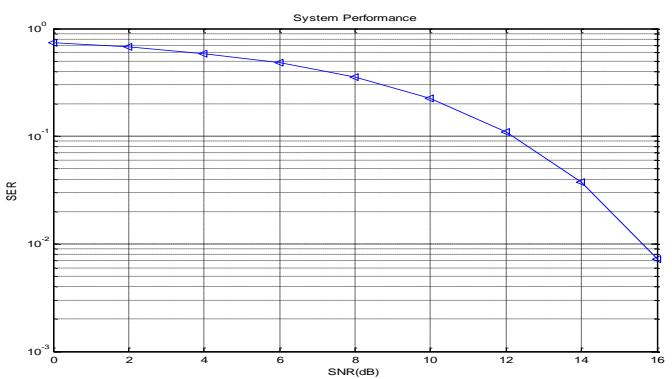

Figure6. Simulated SER Performance For 16-QAM

In this paper, we have demonstrated the application of a 16QAM modulation technique in the OFDM system with a view to reducing the ISI. The experiment has been successful through adding the guard intervals between the consecutive symbols. Although we could not bring the SER reduced to zero our designed system still works properly. The system performance curve demonstrates that it can still be used for DVB-T.

For future work, improvement can be made by coding the original information; over and above, the ISI effect could be further removed by using an equalizer at the receiver end.

\section{REFERENCES}

[1] Mary Ann Ingram and Guillermo Acosta, Smart Antenna Research Laboratory using Matlab, 2000. [E-book] Available: toodoc e-book [Accessed Sep. 23, 2009].

[2] Long Bora and Heau-Jo Kang, "A Study on the Performance of OFDM Transmission Scheme for QAM Data Symbol," 2008 Second International Conference on Future Generation Communication and Networking (FGCN), vol.2, pp.267 - 270, Dec. 2008.

[3] National Instruments, "OFDM and Multi-Channel Communication systems,"2007.[Online]Available:http://zone.ni.com/devzone/cda/tut/p/id 13740 [Accessed Oct. 5, 2009]

[4] Simon Haykin "An introduction to Analog \& Digital communications", 1994, pp. 228-231

[5] K. Takashahi, T. Saba, "A novel symbol synchronization algorithm with reduced influence of ISI for OFDM systems",2001 Global Telecommunication Conference, Globcom "01, IEEE, vol.1, pp.524-528, Nov.2001 USA.

[6] Dukhyun Kim, Stuber, G.L., "Residual ISI cancellation for OFDM with applications to HDTV broadcasting", IEEE Journals on Communication, vol.16, pp.1590-1599, Oct. 1998 